\title{
Scanner Image Analysis to Estimate Leaf Area
}

\author{
Gurjot Kaur \\ School of Electrical Engineering \\ \& IT \\ COAE\&T, PAU \\ Ludhiana, India
}

\author{
Salam Din \\ School of Electrical Engineering \\ \& IT \\ COAE\&T, PAU \\ Ludhiana, India \\ Derminder Singh \\ School of Electrical Engineering \& IT \\ COAE\&T, PAU \\ Ludhiana, India
}

\author{
Amandeep Singh Brar \\ Department of Agronomy \\ COA, PAU \\ Ludhiana, India
}

\begin{abstract}
Several commercial softwares are available for plant leaf area measurement. But no freeware is available for leaf area calculation. This study proposed a freeware for calculating the plant leaf area. It was hypothesized that if the error between the calculated leaf area and the actual leaf area is low then it could be used for agronomical, physiological as well as other researches. Leaves of Brassica species, turmeric, soybean and wheat with different size and shape were selected for the study. The platform used for image processing and leaf area calculation was DOTNET framework at the front end and SQL server at the back end. A digital scanner was utilized to obtain leaf images. The user can browse the scanned image of a leaf in any image format and the freeware automatically convert it into web compatible image format Joint Photographic Group. For color tonal distribution the histogram of images was computed. The colored image was converted into monochrome image by setting the threshold value in the color matrix. In image processing method leaf area was calculated through pixel number statistic. The results were compared with the results of Leaf Area Meter by Disha Infoway India Private Limited and concluded that the error rate between them is less than $\pm 2 \%$ for Brassica species, turmeric, soybean and wheat leaves.
\end{abstract}

\section{General Terms}

User Interface, Digital Image Processing

\section{Keywords}

Leaf Area, Image Processing, C\#.NET, SQL, Freeware, Scanner

\section{INTRODUCTION}

Leaf is an organ of a plant. Monitoring leaf area is important for many physiological and agronomic studies, such as plant growth, photosynthesis and transpiration. Measuring leaf area removed as a result of insect herbivory can be useful for evaluating host-plant resistance, pesticide activity and plantinsect interactions. It is also helpful in evaluating the damage caused by leaf diseases and in analyzing fertilizer deficiency and environmental stress. Leaf area can be calculated by using direct and indirect methods. Direct methods are destructive. They are the most accurate, but they have the disadvantage of being extremely time-consuming and as a result large-scale implementation becomes marginally feasible. Indirect methods have been developed that are nondestructive, inexpensive and fairly rapid [3] but they are not very accurate.

Leaf area meters have been widely adopted for these measurements because of their ease of operation. But they are expensive and measuring leaf area by hand with a sampling grid is time consuming [12]. Also, Leaf area meter cannot handle large leaves. A large leaf has to be cut into smaller pieces and the area for each piece will have to be measured individually so that one can later obtain the area of the whole leaf; however this process can cause measurement errors. Experience has shown that the other methods are tedious, particularly when a large number of leaves are to be measured. Methods involving image processing based on video camera images and computer programs for analysis of these images permit automatic calculation of leaf areas, and of areas lost to herbivores or disease, depending on the computer programs used.

Landis et al (2002) used the public domain software (Image 4.0.2 for Windows) for measuring the surface area of objects in a digital format. The user was required to be familiar with the inbuilt image processing tools of the software to convert an image into monochrome image. Only then one could be able to calculate the leaf surface area. Cunha (2003) used the digital scanner to acquire leaf images with a resolution of 150 dpi. But this system was unable to deal with images having different resolutions. Hernandez-Hernandez et al (2009) examined the accuracy of two leaf area determination methods using digital photographs processed in Matlab and Computer Aided Design (CAD) software. While measuring, leaves should be kept as flat as possible to avoid area measurement errors due to curved leaves.

This study aimed to present an alternative image processing method for estimation of the plant leaf area by using computer programs. These computer programs were developed to process the images automatically having different resolutions. The user only needed to browse the scanned image of a leaf to calculate its area. A unit centimeter square $\left(\mathrm{cm}^{2}\right)$ was selected to convert pixels to a unit of measurement. In image processing method leaf area was calculated through pixel number statistic. Also an image histogram was plotted to represent the tonal distribution in a digital image. It facilitated to check whether an image detail had been lost to blown-out highlights or blacked-out shadows.

\section{MATERIAL AND METHDOLOGY}

Leaf images of Brassica species, turmeric, soybean and wheat with different shapes and sizes were used to validate the proposed method. Leaf area calculated by Leaf Area Meter-by Disha Infoway India Private Limited dependent on HewlettPackard Scan Jet 7400C scanner was considered as standard area. 


\subsection{Existing Commercial Software}

The existing commercial softwares have many limitations. For example, commercial software developed by Disha Infoway India Private Limited was dependent on Hewlett-Packard Scan Jet $7400 \mathrm{C}$ scanner. It was not compatible with any other scanner. It dealt with only black and white bitmap images. Bitmap (.bmp) files are usually not compressed and, therefore, are not well suited for transfer across the Internet. It was unable to handle other image formats like .jpg, .png, .tif, .gif etc.

\subsection{Proposed Freeware}

A freeware is software that is available for use at no monetary cost, but with one or more restricted usage rights such as source code being withheld or redistribution prohibited. For this, the platform used for image processing and leaf area calculation was DOTNET framework at the front end and SQL (Structured Query Language) server at the back end. Microsoft Visual Studio 2010 was used for making the user friendly interface. C\#.NET language was used for writing the source code. SQL Server was used to create backup for the freeware. Leaf's length, width, resolution, area, date and time were saved in the database to maintain the record. A user can go through the record anytime to recover information if the system fails. This freeware is compatible with 32-bit as well as 64-bit processors.

In image processing method, leaf area was calculated through pixel number statistic. The digital images of leaves of Brassica species, turmeric, soybean and wheat with different size and shape were obtained by using a MP Navigator EXCanoScan LiDE 100 scanner. These leaves can be scanned in any form such as colored, sepia, grayscale or black and white. The freeware automatically converted the colored image into black and white image by setting the threshold value in the color matrix. The digital images of leaves obtained can be saved in any image format i.e. .JPG, .BMP, .PNG, .TIF, .GIF etc. The new freeware automatically converted any inputted image format into .JPG format so as to make it webcompatible.

The user only needs to browse the scanned image of a leaf to calculate its area. The leaf area was calculated in centimeter square $\left(\mathrm{cm}^{2}\right)$ using the resolution of the images. The term resolution is often used for a pixel count in digital imaging. Resolution of an image depends upon the size of the pixel. The smaller the size of the pixel, the higher the resolution will be and the clearer the object in the image will be. Images having smaller pixel sizes occupy more space on the disk. Resolution is expressed in Dots per Inch (DPI). After processing, the black pixels of an image were counted as Pixel count .

$$
\text { Leaf Area }(\text { Sq.cm })=\left(\frac{\text { Pixel }_{\text {count }}}{d p i^{2}}\right) * 6.4516
$$

The error formula adopted was presented by Ali et al (2012), which was:

$$
\text { Error Rate }(\%)=\left[\frac{A 1-A 2}{A 2}\right] * 100
$$

Where: A1 is the estimated area by the proposed method, A2 is the actual area estimated by Leaf Area Meter-by Disha Infoway India Private Limited. Calculating error rate or percentage error allows comparing an estimate to an exact value. The percentage error gives the difference between the approximate and exact values as a percentage of the exact value and can help to see how close the estimate was to a real value.

Also a histogram of an image was plotted to check whether an image detail had been lost to blown-out highlights or blackedout shadows. An image histogram is a type of histogram that serves as a graphical representation of the tonal distribution in a digital image. It plots the number of pixels for each tonal value. By looking at the histogram for a specific image a viewer will be able to judge the entire tonal distribution at a glance.

\section{RESULTS AND DISCUSSIONS}

All the graphs presented in this paper were obtained after executing the proposed freeware and commercial software.

\subsection{Comparison of Leaf Area Calculated by Commercial and Proposed Freeware}

To test the performance of the new proposed freeware, leaves of Brassica species, turmeric, soybean and wheat with different size and shape were scanned by using HewlettPackard Scan Jet 7400C scanner. Area of same set of scanned leaves was calculated by using Leaf Area Meter-by Disha Infoway India Private Limited as well as proposed freeware. Leaf area calculated by Leaf Area Meter dependent on Hewlett-Packard Scan Jet 7400C scanner was considered as standard area. The results of proposed freeware and Leaf Area Meter-by Disha Infoway India Private Limited were compared and found that the relative error value for Brassica species, turmeric, soybean and wheat leaves respectively ranges between $\pm 2 \%$.

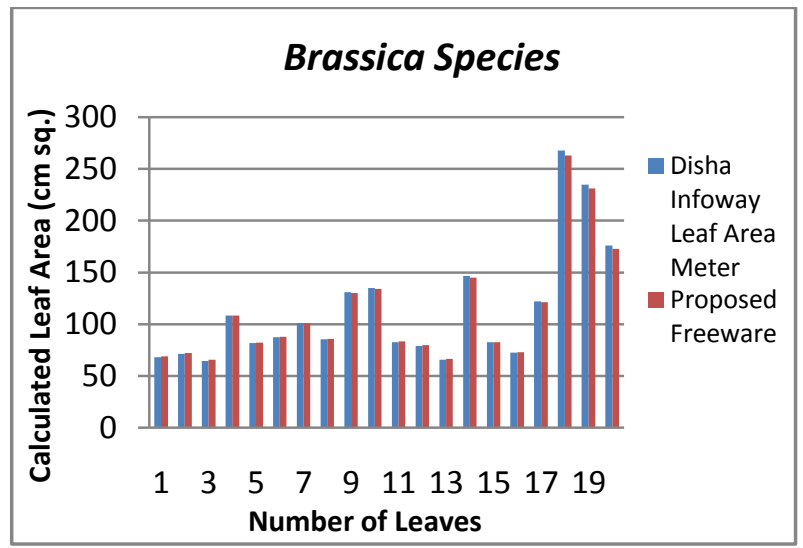

Fig 3.1 Comparison of Leaf Area of Brassica species Leaves Calculated by Commercial and Proposed Software

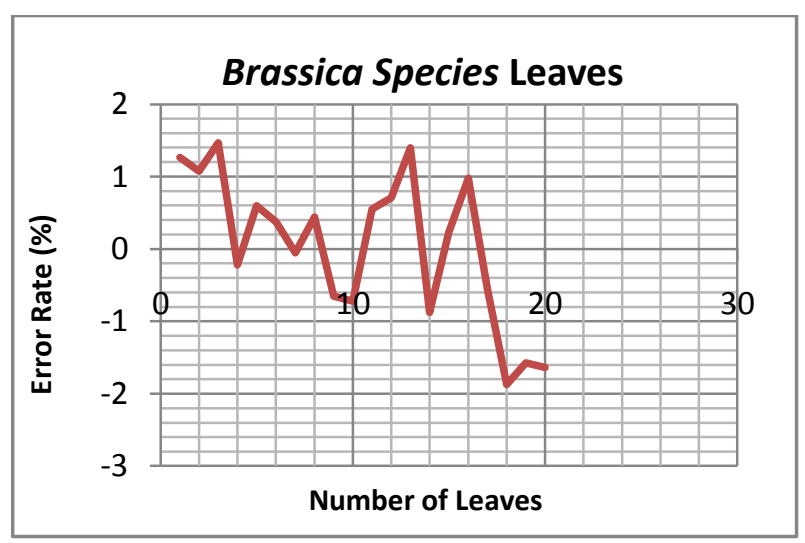

Fig 3.2 Range of Error Rate among Brassica species Leaves 


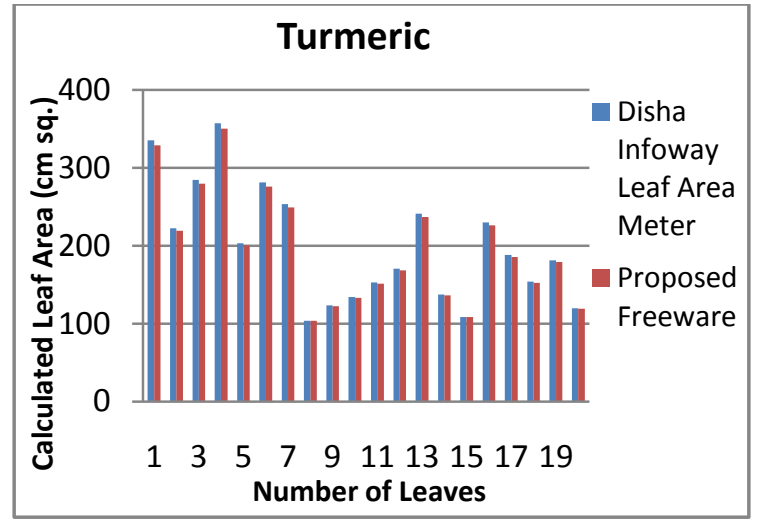

Fig 3.3 Comparison of Leaf Area of Turmeric Leaves Calculated by Commercial and Proposed Software

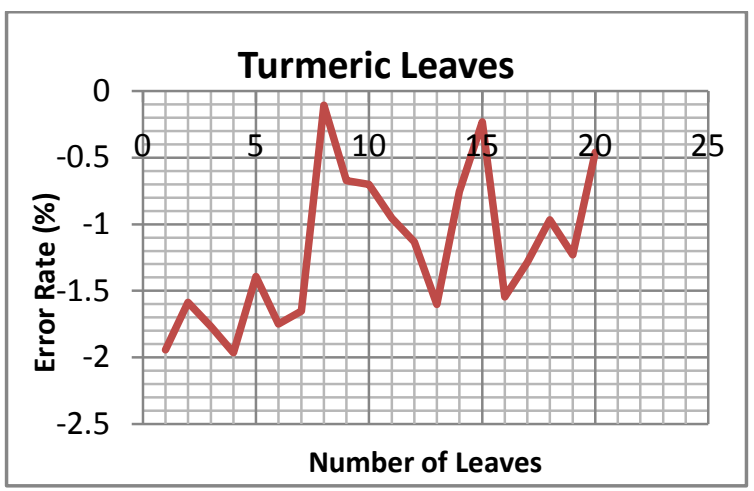

Fig 3.4 Range of Error Rate among Turmeric Leaves

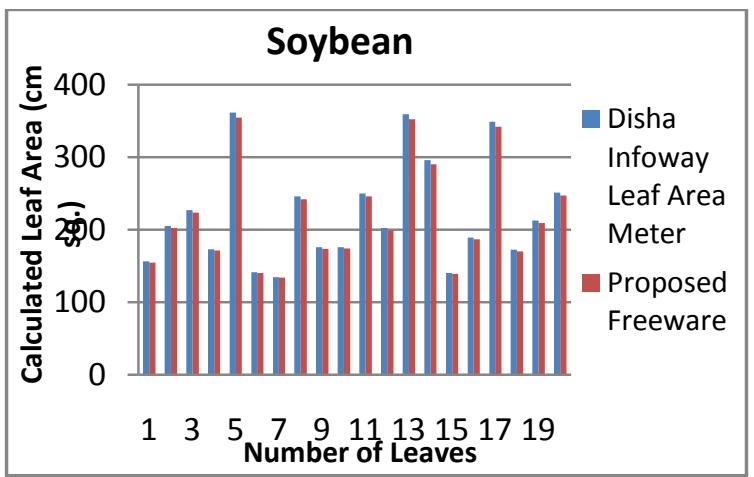

Fig 3.5 Comparison of Leaf Area of Soybean Leaves Calculated by Commercial and Proposed Software

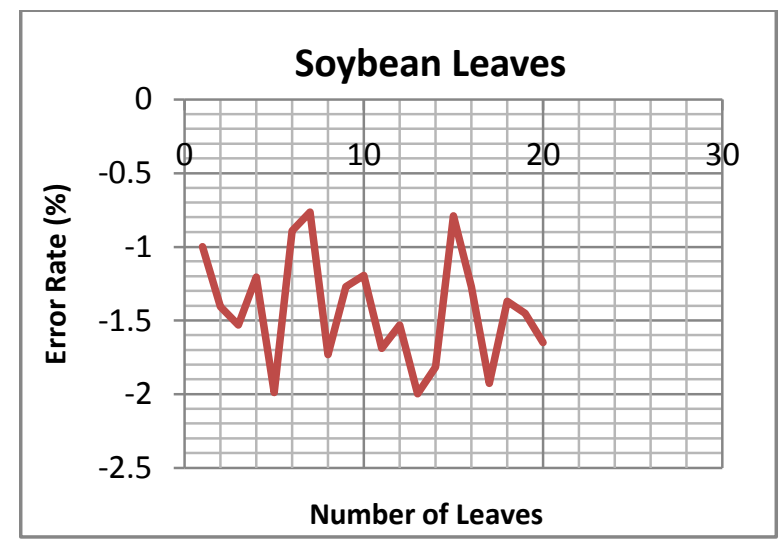

Fig 3.6 Range of Error Rate among Soyabean Leaves

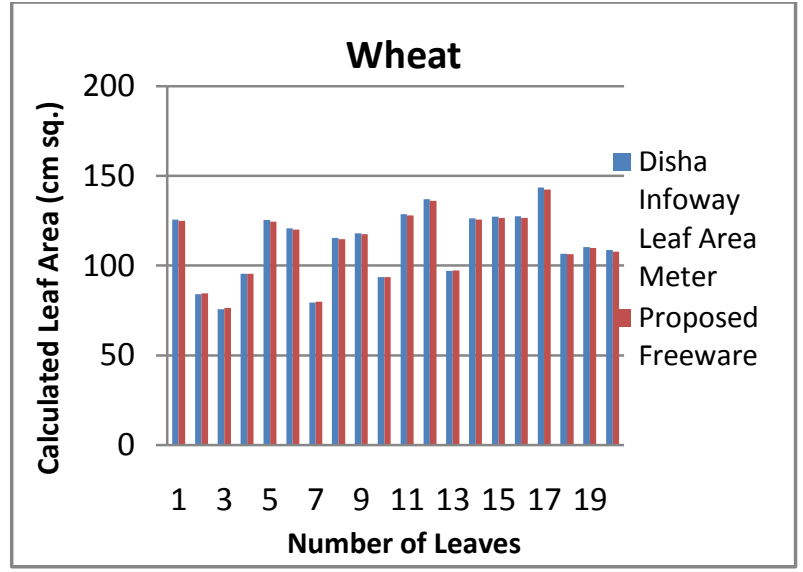

Fig 3.7 Comparison of Leaf Area of Wheat Leaves Calculated by Commercial and Proposed Software

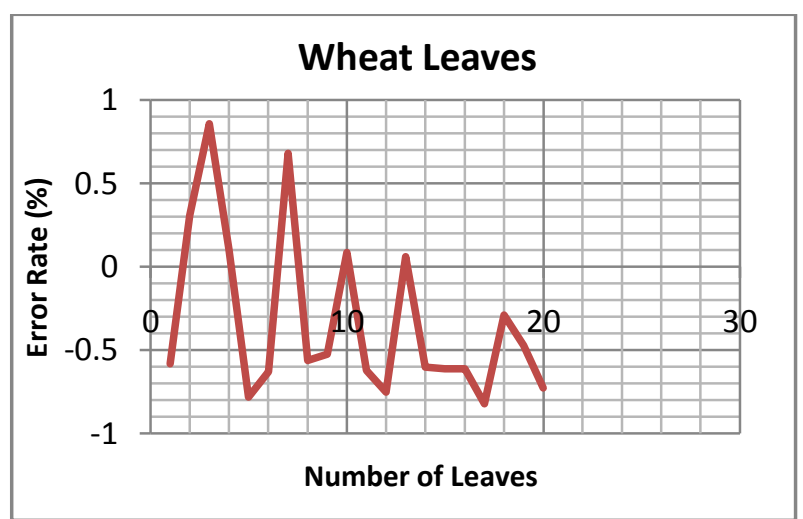

Fig 3.8 Range of Error Rate among Wheat Leaves

\subsection{Comparison of Leaf Area of Leaves} Scanned by HP and Canon Scanner

A sample of 4 leaves was scanned by using Hewlett-Packard (HP) Scan Jet 7400C scanner and MP Navigator EXCanoScan LiDE 100 scanner with resolution of 150dpi. Figure 3.9 depicted the comparison of leaf area of leaves scanned by two different scanners. The leaf area was calculated using new proposed freeware and discovered that relative error rate for images scanned by $\mathrm{HP}$ and Canon scanner was less than $\pm 2 \%$ and $\pm 1.6 \%$ respectively as shown in Figure 3.10. This means that the new proposed freeware is independent of the scanner used.

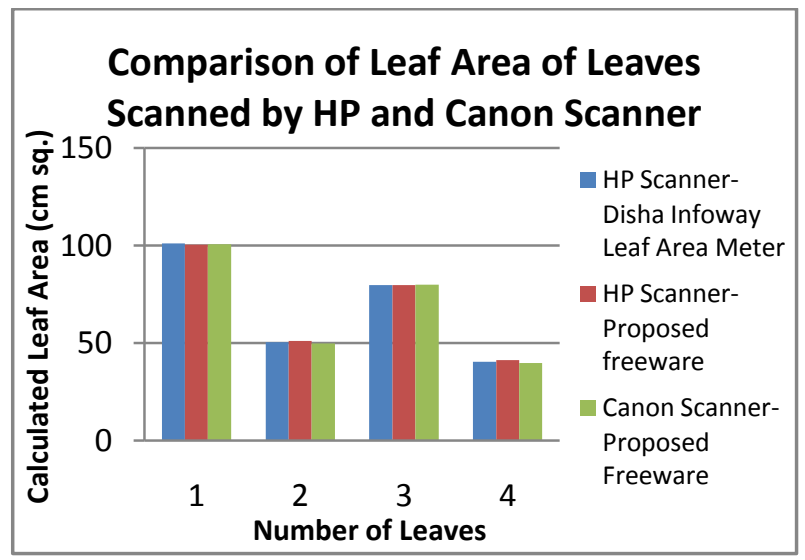

Fig 4.9: Comparison of Leaf Area of Leaves Scanned by Two Different Scanners 


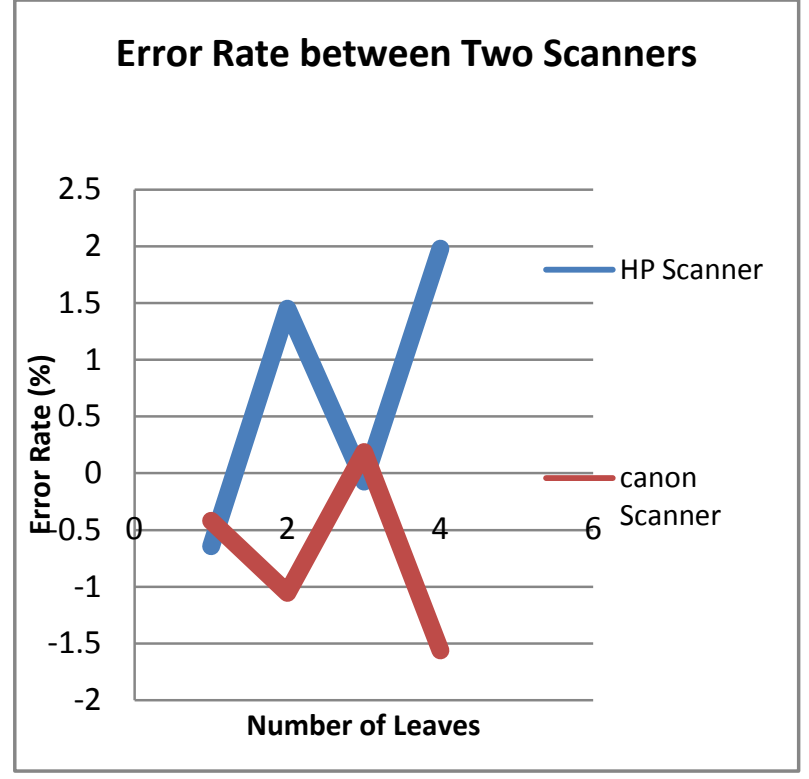

Fig 3.10 Range of Error Rate of Leaves Scanned by Two Different Scanners

\subsection{Comparison of Leaf Area of Leaves} Scanned at 150 dpi and 300 dpi Resolutions

A sample of 5 leaves was scanned by using Hewlett-Packard (HP) Scan Jet 7400C scanner at resolution of 150dpi and 300dpi in .bmp format. The scanner resolution was $2400 \times 2400 \mathrm{dpi}$ and color-depth was 48 bit. The color-depth of .bmp image was 1-bit, and the color-depth of scanner was 48bit. But still it output only in 1-bit color by performing internal processing to select the best possible choice out of the colors available in the increased palette. The leaf area was calculated using new proposed freeware as portrayed in Figure 4.11 and 4.12 and it was found that the error rate between the areas of scanned leaves ranged between 0 to $2 \%$ for 150 dpi and 0 to $1.5 \%$ for 300 dpi as depicted in Figure 4.13 and 4.14. Hence, more accurate results were achieved at 300 dpi.

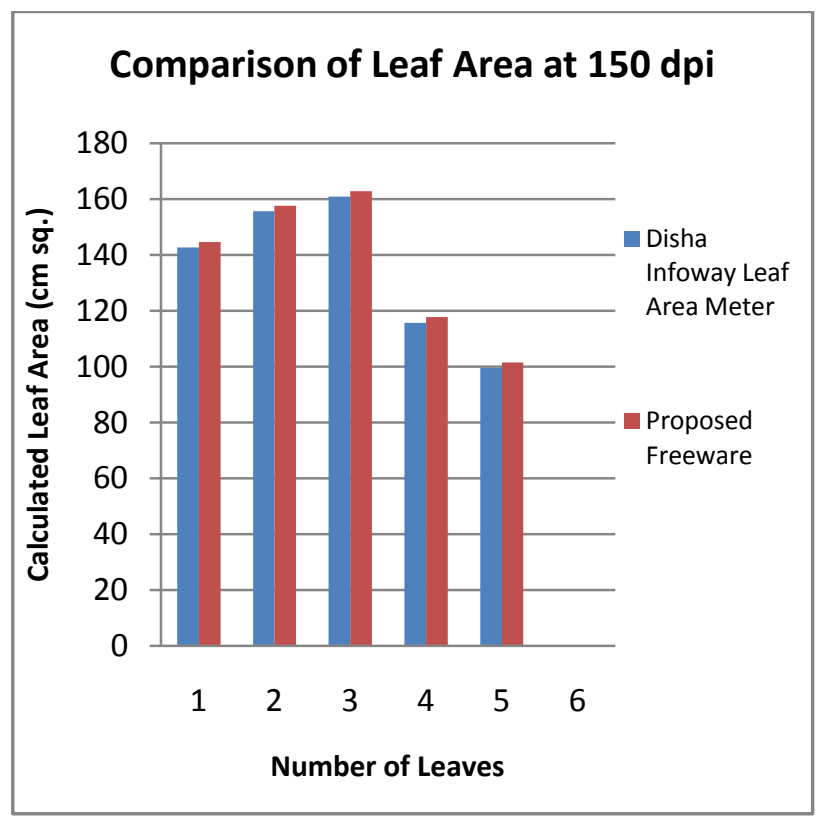

Fig 4.11: Comparison of Leaf Area at 150 dpi Resolution

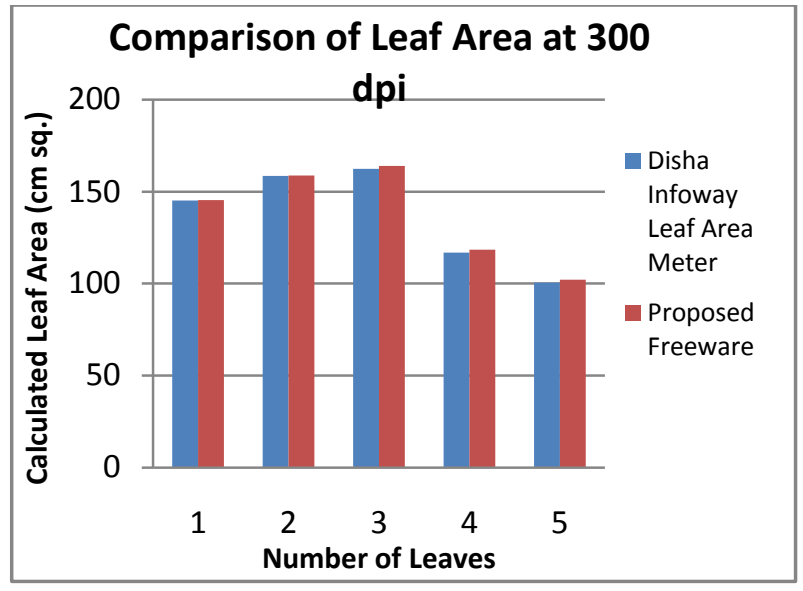

Fig 4.12 Comparison of Leaf Area at 300 dpi Resolution

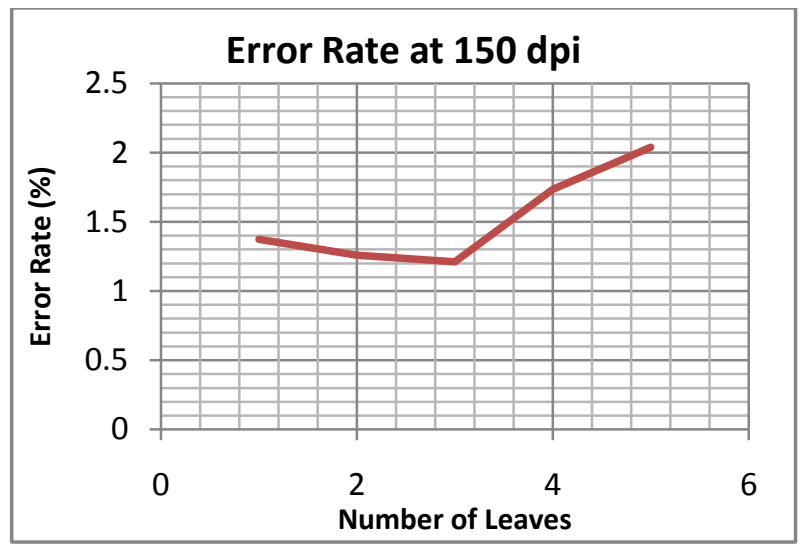

Fig 4.13 Range of Error Rate at 150 dpi Resolution

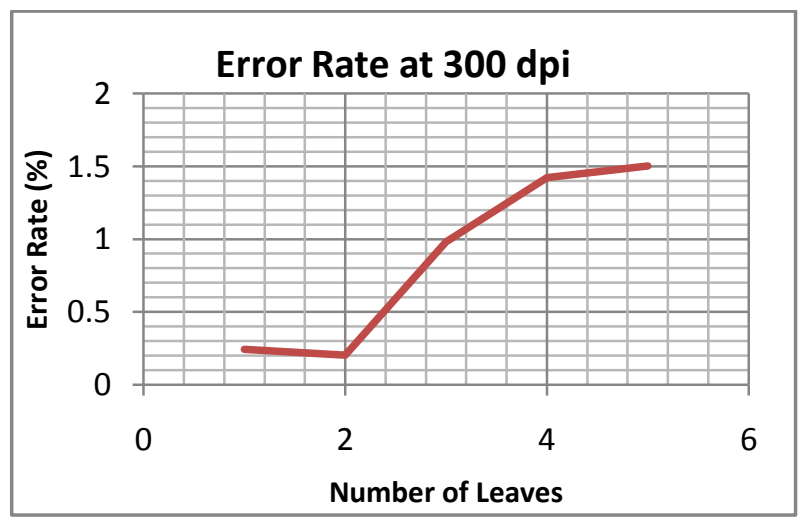

Fig 4.14 Range of Error Rate at 300 dpi Resolution

\subsection{Comparison of Leaf Area of Scanned} Leaves Using Different Image File Formats

A sample of 5 leaves was scanned by using Hewlett-Packard (HP) Scan Jet 7400C scanner with 150 dpi resolution. Each Leaf was scanned by choosing the option 'Black and White Bitmap' and saved in .bmp and tif formats. Same set of leaves were scanned by using 'True Color' option and saved in .bmp, .tif and .jpg formats. The position of a leaf was kept stable. Now Leaf Area Meter provided by Disha Infoway India Private Limited processes only black and white BMP images. But the Proposed freeware accepts images stored in any format and converts them into JPG format for further processing. Figure 3.15 and 3.17 compares the area of monochrome and colored images respectively. While processing monochrome images, the error rate in BMP and 
TIF format ranges from 0 to $2 \%$ as depicted in Figure 3.16. In case of colored images, the error rate for TIF, BMP and JPG format ranged from 0 to $-1.5 \%$ as portrayed in Figure 3.18. It was concluded that the average error rate was minimum in colored TIF format and maximum in black and white BMP format.

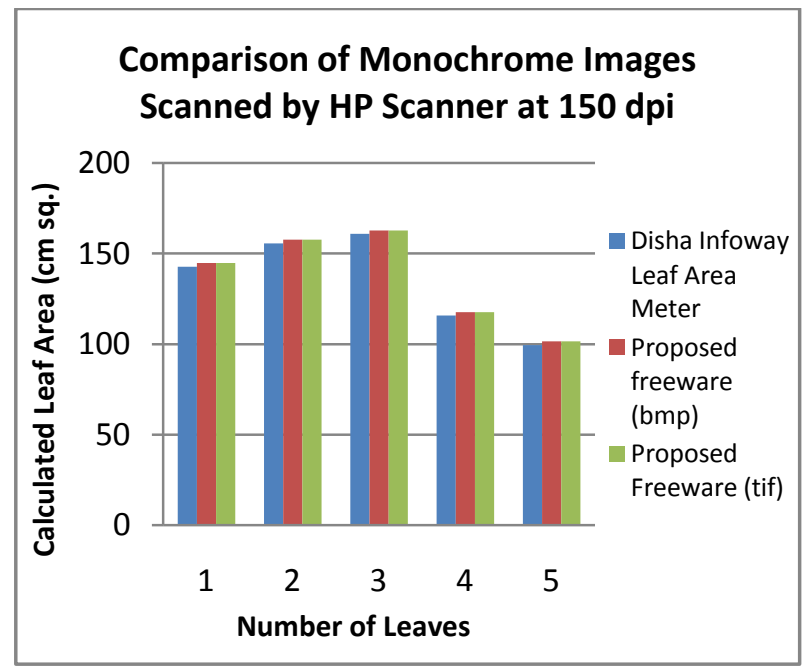

Fig 3.15 Comparison of Leaf Area of Monochrome Images Saved in Different Formats

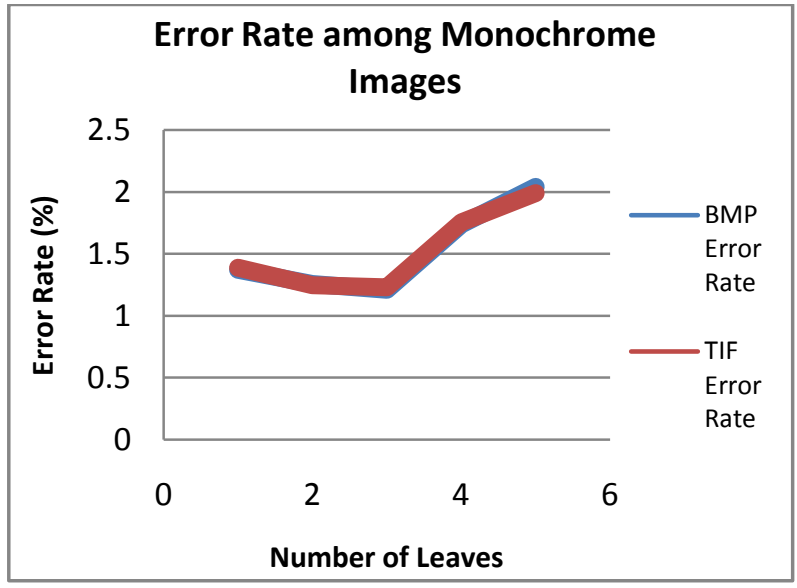

Fig 3.16 Range of Error Rate among Monochrome Images Saved in Different Formats

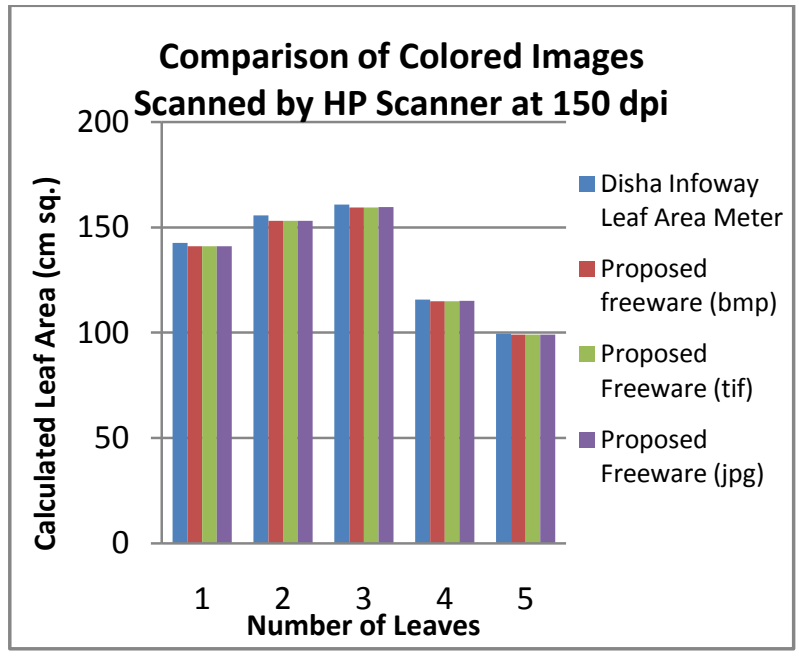

Fig 3.17 Comparison of Leaf Area of Colored Images Saved in Different Formats

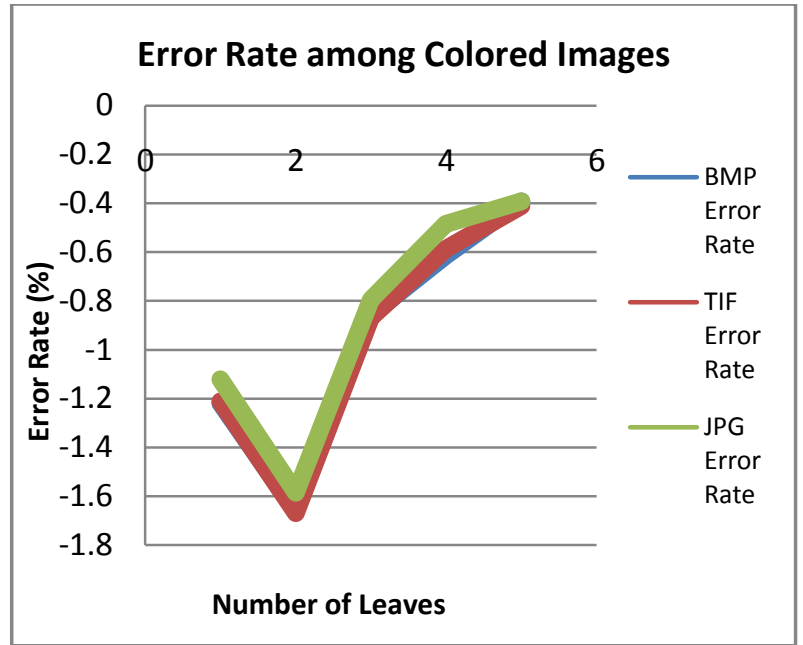

Fig 3.18 Range of Error Rate among Colored Images Saved in Different Formats

\section{CONCLUSION}

A new freeware developed using image processing approach can be considered as an inexpensive, scanner independent methodology that is good enough to calculate the area of scanned leaves with different resolutions. The error rate seems to be within the limit with only \pm 2 percent variation. This variation may be due to the different size, shape, health, color nutrient differences as well as insect-pest attack on leaves. While scanning, leaves should be kept as flat as possible to avoid area measurement errors due to curved leaves.

\section{REFERENCES}

[1] Adiga B S, Deepak N and Nandagopalan S (2009) A Universal Model for Content-Based Image Retrieval. Int J Elec Comput Eng 4:249-252.

[2] Ali M M, Al-Ani A, Eamus D and Tan D K Y (2012) A new image-processing based technique for measuring leaf dimensions. American-Eurasian J Agric \& Environ Sci 12:1588-1594.

[3] Baret F, Coppin P, Fleck S, Jonckheere I, Muys B, Nackaerts K and Weiss M (2004) Review of methods for in situ leaf area index determination Part I. Theories, sensors and hemispherical photography. Agr Forest Meteorol 121:19-35.

[4] Bodhe S K and Patil S B (2011) Image processing method to measure sugarcane leaf area. Int J Eng Tech 3:6394-6400

[5] Braga R A, Horgan G W, Marcon M, Mariano K, Paglis C M and Scalco M S (2011) Estimation of total leaf area in perennial plants using image analysis. Rev Bras Eng Agric Ambient 15:96-101.

[6] Camargo A and Smith J S (2009) An image-processing based algorithm to automatically identify plant disease visual symptoms. Biosyst Eng 102:09-21.

[7] Chen J M, Miller J R and Zhang Y (2005) Determining digital hemispherical photograph exposure for leaf area index estimation. Agr Forest Meteorol 133:166-181.

[8] Cristofori V de-Gyves E M, Fallovo C, Fanasca S, Rea R and Rivera C M (2008) Leaf Area Estimation Model for Small Fruits from Linear Measurements. Hortsci 43:2263-2267. 
[9] Cunha J B (2003) Application of image processing techniques in the characterization of plant leafs. IEEE T Ind Electron 1:612-616.

[10] Femat-Diaz A, Herrera-Ruiz G, Huerta-Manzanilla E, Rico-Gracia E and Vargas-Vazquez D (2011) Scanner image methodology (SIM) to measure dimensions of leaves for agronomical applications. Afr J Biotechnol 10:1840-1847.

[11] Hernandez-Hernandez F, Herrera-Ruiz G, Rico-Gracia E and Soto-Zarazua G M (2009) Two new Methods for the Estimation of Leaf Area using Digital Photography. Int $J$ Agric Biol 11:397-400.

[12] Landis D A, Isaacs R and O'Neal M E (2002) An Inexpensive, Accurate Method for Measuring Leaf Area and Defoliation Through Digital Image Analysis. J Econ Entomol 95:1190-1194.

[13] Lu C, Ren H, Shen Y and Zhang Y (2010) Leaf Area Measurement Based on Image Processing. Proc of International Conference on Measuring Technology and Mechatronics Automation (ICMTMA) Vol 2, pp 580-582, Changsha City, China.

[14] Phattaralerphong J, Sathornkich J and Sinoquet H (2006) A photographic gap fraction method for estimating leaf area of isolated trees: assessment with 3D digitized plants. Tree Physiol 26:1123-1136.

[15] Singh Y and Vij K (2012) Enhancement of Images Using Histogram Processing Techniques. Int J Comp Tech Appl 2:309-313. 